\title{
Regularity of Constraints in the Minkowski Space Yang-Mills Theory*
}

\section{Jędrzej Śniatycki}

Department of Mathematics and Statistics, University of Calgary, Calgary, Alberta, Canada

Received January 16, 1991; in revised form April 24, 1991

\begin{abstract}
For Yang-Mills theory in the Minkowski space it is proved that the constraint set is a smooth submanifold of the phase space consisting of square integrable Cauchy data.
\end{abstract}

\section{Introduction}

Yang-Mills equations can be rewritten as the evolution equations for the Cauchy data supplemented by the constraint condition. In the temporal gauge the evolution equations are

$$
\begin{gathered}
\dot{\mathbf{A}}=\mathbf{E}, \\
\dot{\mathbf{E}}=\operatorname{div} \mathbf{F}+[\mathbf{A} ; \mathbf{F}],
\end{gathered}
$$

where

$$
\mathbf{F}=d \mathbf{A}+[\mathbf{A}, \mathbf{A}]
$$

is the field strength of $\mathbf{A}$, and

$$
[\mathbf{A} ; \mathbf{F}]^{a}=c_{b c}^{a} \mathbf{A}_{i}^{b} \mathbf{F}^{c i}
$$

is the Lie bracket in the Lie algebra $g$ of the structure group $G$ of the theory, followed by the contraction in the spatial indices. The constraint equation is

$$
\operatorname{div} \mathbf{E}+[\mathbf{A} ; \mathbf{E}]=0 .
$$

In order to have an understanding of the theory sufficient for a subsequent quantization, we need to know the spaces which admit existence and uniqueness theorems for the system (1), (2), and (5), and the structure of the solution set of the constraint equation [since Eq.(5) is non-linear its solution set may have singularities].

\footnotetext{
* Partially supported by the NSERC Operating Grant No. A8091
} 
For Yang-Mills theory in Minkowski space the existence and uniqueness theorems were obtained by Segal [1], Ginibre and Velo [2], and Eardley and Moncrief $[3,4]$. They have shown that, for Cauchy data $(\mathbf{A}(0), \mathbf{E}(0))$ in the Sobolev space $H^{k}\left(\mathbb{R}^{3}\right) \times H^{k-1}\left(\mathbb{R}^{3}\right), k \geqq 2$, which satisfy the constraint equation, there exists a global solution (A(t), E(t)) of Eqs. (1), (2), and (5). The regularity of constraints in weighted Sobolev spaces $M_{s, \delta}^{p}$ with $p>3,1 \leqq \delta<2-3 / p$, and $s \geqq 2$, was proved by Moncrief [5].

Since the regularity theorems hold in different spaces than the existence and uniqueness theorems we do not have a complete classical theory. The aim of this paper is to prove the regularity of the constraint set in the space $H^{2}\left(\mathbb{R}^{3}\right) \times H^{1}\left(\mathbb{R}^{3}\right)$ for which we already have the existence and uniqueness theorem. The proof presented here, based on the standard techniques of perturbations of linear operators, is somewhat simpler than in [5].

The Second Noether Theorem implies that the invariance of a field theory under a localizable group $\mathscr{G}$ of transformations leads to constraints, given by the vanishing of the equivariant momentum map $\mathbf{J}$ of the action of $\mathscr{G}$ in the phase space of the theory, cf. [6]. In Yang-Mills theory the group $\mathscr{G}$ consists of the gauge transformations which can be identified with sections of the group bundle $P[G]$ associated to the principal fibre bundle $P$ over $\mathbb{R}^{3}$ with structure group $G$ of the theory. Infinitesimal gauge transformations are sections of the adjoint bundle $P[\mathrm{~g}]$. The action of a section $u$ of $P[\mathfrak{g}]$ on Cauchy data $(\mathbf{A}, \mathbf{E})$ is

$$
(\mathbf{A}, \mathbf{E}) \rightarrow(\mathbf{A}+d u+[\mathbf{A}, u], \mathbf{E}+[\mathbf{E}, u]) .
$$

The value of the momentum map $\mathbf{J}$ on an infinitesimal gauge transformation $u$ is given by

$$
\langle\mathbf{J}(\mathbf{A}, \mathbf{E}) \mid u\rangle=\int_{\mathbb{R}^{3}}(\mathbf{E} \mid d u+[\mathbf{A}, u]) d_{3} x,
$$

where the pointwise evaluation (|) uses the scalar products in $\mathbb{R}^{3}$ and in $\mathfrak{g}$. Hence, the zero level of $\mathbf{J}$ coincides with the solution set of the constraint equation (5).

A study of the structure of the constraint set in Yang-Mills theory, in terms of the corresponding equivariant momentum map $\mathbf{J}$, has been performed by Arms [7], and Arms, Marsden, and Moncrief [8]. Under technical assumptions, depending on the structure of the space of Cauchy data, it has been shown that $\mathbf{J}^{-1}(0)$ has at most quadratic singularities, with a regular stratum consisting of the Cauchy data (A, E) with no infinitesimal symmetry in $\mathscr{G},[8]$.

Equation (6) shows that $u$ is an infinitesimal symmetry of $(\mathbf{A}, \mathbf{E})$ if and only if

$$
d u+[\mathbf{A}, u]=0 \text { and }[\mathbf{E}, u]=0,
$$

which implies that the square of the norm of $u$ is constant,

$$
d(u \mid u)=0 \text {. }
$$

In the Minkowski space Yang-Mills theory an infinitesimal symmetry $u$ is localizable if and only if $u$ vanishes at infinity, [6]. Hence, Eq. (9) implies that $u=0$. Thus, no square integrable Cauchy data $(\mathbf{A}, \mathbf{E})$ admit a non-zero infinitesimal localizable gauge symmetry. If the Arms, Moncrief, Marsden Theorem is applicable to this case, we can infer that the constraint set $\mathbf{J}^{-1}(0)$ is regular, that is every point of $\mathbf{J}^{-1}(0)$ is a regular point. This guaranties that $\mathbf{J}^{-1}(0)$ is a submanifold of the space of Cauchy data, and that the Marsden-Weinstein reduced phase space $\mathbf{J}^{-1}(0) / \mathscr{G}$ is a symplectic manifold, [9]. 
Thus, it would suffice to verify the assumptions of the Arms, Marsden, Moncrief Theorem in order to conclude that in the Minkowski space Yang-Mills theory the constraint set is regular. However, it is simpler to prove the regularity of $\mathbf{J}^{-1}(0)$ directly.

\section{Regularity of $\mathbf{J}^{-1}(0)$}

For each pair $(k, m)$ of non-negative integers, we denote by $\Omega_{m}^{k}$ the space of $\mathfrak{g}$-valued $m$-forms with coefficients in the Sobolev space $H^{k}\left(\mathbb{R}^{3}\right)$. The product structure in the principal fibre bundle $P$ enables us to interpret $H^{k}\left(\mathbb{R}^{3}\right)$ sections of the adjoint bundle $P[g]$ as elements of $\Omega_{0}^{k}$. Similarly, connections in $P$ can be identified with $\mathfrak{g}$-valued 1 -forms.

It follows from the results of Eardley and Moncrief, $[3,4]$, that, for every set of Cauchy data $(\mathbf{A}, \mathbf{E})$ in $\Omega_{1}^{2} \times \Omega_{1}^{1}$ satisfying the constraint equation, there exists a global solution of Yang-Mills equations. For $(\mathbf{A}, \mathbf{E})$ in $\Omega_{1}^{2} \times \Omega_{1}^{1}$

$$
\mathbf{J}(\mathbf{A}, \mathbf{E})=\operatorname{div} \mathbf{E}+[\mathbf{A} ; \mathbf{E}]
$$

is contained in $\Omega_{0}^{0}$. Hence, in order to show that $(\mathbf{A}, \mathbf{E}) \in \Omega_{1}^{2} \times \Omega_{1}^{1}$ is a regular point of $\mathbf{J}$, one needs to show that

$$
d \mathbf{J}(\mathbf{A}, \mathbf{E}): \Omega_{1}^{2} \times \Omega_{1}^{1} \rightarrow \Omega_{0}^{0}:(\mathbf{w}, \mathbf{v}) \mapsto \operatorname{div} \mathbf{v}+[\mathbf{A} ; \mathbf{v}]+[\mathbf{w} ; \mathbf{E}]
$$

is surjective and has splitting kernel. Actually, it suffices to show that, for each $\mathbf{A} \in \Omega_{1}^{2}$, the map

$$
\operatorname{Div}_{\mathbf{A}}: \Omega_{1}^{1} \rightarrow \Omega_{0}^{0}: \mathbf{v} \mapsto \operatorname{Div}_{\mathbf{A}}(\mathbf{v})=\operatorname{div} \mathbf{v}+[\mathbf{A} ; \mathbf{v}]
$$

is surjective and has splitting kernel. Since $\operatorname{Div}_{\mathbf{A}}$ is continuous map between Hilbert spaces it follows that its kernel is closed subspace and it has a closed orthogonal complement. Hence it remains to show that Div $_{\mathbf{A}}$ is surjective.

For every integer $k \geqq 1$, each 1 -form $\mathbf{v} \in \Omega_{1}^{k-1}$ can be uniquely decomposed into the longitudinal and the transverse parts

$$
\mathbf{v}=\mathbf{v}^{L}+\mathbf{v}^{T}
$$

where

$$
\mathbf{v}^{L}=d u
$$

for some $u \in \Omega_{0}^{k}$, and

$$
\operatorname{div} \mathbf{v}^{T}=0 .
$$

We denote by $\Omega_{1}^{k-1^{T}}$ and $\Omega_{1}^{k-1^{L}}$ the spaces of transverse and longitudinal forms in $\Omega_{1}^{k-1}$, respectively. They are closed subspaces of $\Omega_{1}^{k-1}$, and

$$
\Omega_{1}^{k-1}=\Omega_{1}^{k-1^{T}} \oplus \Omega_{1}^{k-1^{L}} .
$$

We denote by $D_{\mathrm{A}}$ the operator of covariant differentiation of sections of $P[\mathrm{~g}]$,

$$
D_{\mathbf{A}} u=d u+[\mathbf{A}, u]
$$

and consider it as an unbounded linear operator from $\Omega_{0}^{0}$ to $\Omega_{1}^{0}$ with domain $\Omega_{0}^{1}$. For every $u \in \operatorname{domain} D_{\mathbf{A}}$ and every $\mathbf{v} \in \Omega_{1}^{1}$,

$$
\left(D_{\mathbf{A}}(u) \mid \mathbf{v}\right)=-\left(u \mid \operatorname{Div}_{\mathbf{A}}(\mathbf{v})\right)
$$


Hence, the negative of the adjoint $D_{\mathbf{A}}^{*}$ of $D_{\mathbf{A}}$ is an extension of $\operatorname{Div}_{\mathbf{A}}$,

$$
\operatorname{Div}_{\mathbf{A}} \cong-D_{\mathbf{A}}^{*} \text {. }
$$

Since, for $u \in \Omega_{0}^{0}$, $\operatorname{div}(d u) \in \Omega_{0}^{1}$ implies that $u \in \Omega_{0}^{2}$, so that $d u \in \Omega_{1}^{1}$, it follows that

$$
\operatorname{domain}\left(D_{\mathbf{A}}^{*}\right)=\Omega_{1}^{0^{T}} \oplus \Omega_{1}^{1^{L}} \text {. }
$$

A closed operator $T$ between Banach spaces is said to be semi-Fredholm if range $T$ is closed, and at least one of $\operatorname{dim}(\operatorname{ker} T)$ and $\operatorname{codim}(\operatorname{range} T)$ is finite. An operator $S$ is said to be $T$-compact if domain $T \subseteq$ domain $S$, and, for each sequence $\left\{u_{n}\right\} \subset$ domain $T$ with $\left\{u_{n}\right\}$ and $\left\{T u_{n}\right\}$ bounded, the sequence $\left\{S u_{n}\right\}$ has a convergent subsequence. If $T$ is a semi-Fredholm operator and $S$ is $T$-compact, then $T+S$ is semi-Fredholm, for details see [10].

Since $\Omega_{1}^{0^{L}}=$ range $d$ is closed in $\Omega_{1}^{0}$, and $\operatorname{ker} d=0$, it follows that $d$ is semiFredholm operator from $\Omega_{0}^{0}$ to $\Omega_{1}^{0}$. We want to show that, for a given $\mathbf{A} \in \Omega_{1}^{2}, D_{\mathbf{A}}$ is semi-Fredholm. Let $S: \Omega_{0}^{0} \rightarrow \Omega_{1}^{0}$ be the mapping defined by $S u=[\mathbf{A}, u]$. Then $D_{\mathbf{A}}=d+S$ is semi-Fredholm provided $S$ is $d$-compact.

Consider a bounded sequence $u_{n}$ in $\Omega_{0}^{0}$ such that $d u_{n}$ is bounded in $\Omega_{1}^{0}$, which implies that $u_{n} \in \Omega_{0}^{1}$. Since $u_{n}$ and $\mathbf{A}$ are square integrable with their first derivatives, it follows that they are in $L^{4}\left(\mathbb{R}^{3}\right)$, and their squares are in $L^{2}\left(\mathbb{R}^{3}\right)$. Let $M$ denote the $L^{4}\left(\mathbb{R}^{3}\right)$ bound for the sequence $\left\{u_{n}\right\}$. Then,

$$
\left\|\left(u_{n}-u_{m}\right)^{2}\right\|_{L^{2}\left(\mathbb{R}^{3}\right)} \leqq 4 M \text {. }
$$

Moreover, for any a bounded domain $U$ in $\mathbb{R}^{3}$ with complement $U^{\prime}=\mathbb{R}^{3}-U$,

$$
\begin{aligned}
\left\|S\left(u_{n}-u_{m}\right)\right\|^{2} & =\int_{\mathbb{R}^{3}}\left|\left[\mathbf{A}(x), u_{n}(x)-u_{m}(x)\right]\right|^{2} d_{3} x \\
& =\int_{U}\left|\left[\mathbf{A}(x), u_{n}(x)-u_{m}(x)\right]\right|^{2} d_{3} x+\int_{U^{\prime}}\left|\left[\mathbf{A}(x), u_{n}(x)-u_{m}(x)\right]\right|^{2} d_{3} x \\
& \leqq \int_{U}|\mathbf{A}(x)|^{2} \cdot\left|u_{n}(x)-u_{m}(x)\right|^{2} d_{3} x+\int_{U^{\prime}}|\mathbf{A}(x)|^{2} \cdot\left|u_{n}(x)-u_{m}(x)\right|^{2} d_{3} x \\
& \leqq\left\|\mathbf{A}^{2}\right\|_{L^{2}(U)} \cdot\left\|\left(u_{n}-u_{m}\right)^{2}\right\|_{L^{2}(U)}+\left\|\mathbf{A}^{2}\right\|_{L^{2}\left(U^{\prime}\right)} \cdot\left\|\left(u_{n}-u_{m}\right)^{2}\right\|_{L^{2}\left(U^{\prime}\right)} \\
& \leqq\left\|\mathbf{A}^{2}\right\|_{L^{2}\left(\mathbb{R}^{3}\right)} \cdot\left\|\left(u_{n}-u_{m}\right)^{2}\right\|_{L^{2}(U)}+\left\|\mathbf{A}^{2}\right\|_{L^{2}\left(U^{\prime}\right)} \cdot\left\|\left(u_{n}-u_{m}\right)^{2}\right\|_{L^{2}\left(\mathbb{R}^{3}\right)} \\
& \leqq\left\|\mathbf{A}^{2}\right\|_{L^{2}\left(\mathbb{R}^{3}\right)} \cdot\left\|\left(u_{n}-u_{m}\right)\right\|_{L^{4}(U)}^{4}+4 M\left\|\mathbf{A}^{2}\right\|_{L^{2}\left(U^{\prime}\right)} .
\end{aligned}
$$

Since $\left\|\mathbf{A}^{2}\right\|_{L^{2}\left(\mathbb{R}^{3}\right)}$ is the limit of $\left\|\mathbf{A}^{2}\right\|_{L^{2}(U)}$ as $U$ tends to $\mathbb{R}^{3}$, for every $\varepsilon>0$, there exists an open bounded set $U \subseteq \mathbb{R}^{3}$ such that

$$
4 M\left\|\mathbf{A}^{2}\right\|_{L^{2}\left(U^{\prime}\right)}<\varepsilon / 2 \text {. }
$$

For a bounded domain $U$ in $\mathbb{R}^{3}$, the Rellich-Kondrachov Theorem implies that the embedding $H^{1}\left(\mathbb{R}^{3}\right) \rightarrow L^{4}(U)$ is compact, see [11]. Hence the sequence $u_{n} \mid U$ has a subsequence convergent in $L^{4}(U)$ and, without loss of generality, we can assume that $u_{n} \mid U$ is convergent in $L^{4}(U)$. Therefore, there exists $N>0$ such that

$$
\left\|\mathbf{A}^{2}\right\|_{L^{2}\left(\mathbb{R}^{3}\right)} \cdot\left\|\left(u_{n}-u_{m}\right)\right\|_{L^{4}(U)}^{4}<\varepsilon / 2
$$

for all $n, m>N$. Hence, $\left\|S\left(u_{n}-u_{m}\right)\right\|^{2}<\varepsilon$ for all $n, m>N$, which implies that the sequence $S u_{n}=\left[\mathbf{A}, u_{n}\right]$ converges in $\Omega_{1}^{0}$. This implies that $S$ is $d$-compact so that $D_{\mathbf{A}}$ is semi-Fredholm for all $\mathrm{A} \in \Omega_{1}^{2}$. Hence, $D_{\mathrm{A}}$ has closed range.

A vector $\mathbf{v} \in \Omega_{1}^{0}$ is orthogonal to range $D_{\mathbf{A}}$ if and only if

$$
\left(\mathbf{v} \mid D_{\mathbf{A}} u\right)=0 \forall \quad u \in \text { domain } D_{\mathbf{A}} \text {. }
$$


This implies that $\mathbf{v} \in$ domain $D_{\mathbf{A}}^{*}$ and

$$
\left(D_{\mathbf{A}}^{*} \mathbf{v} \mid u\right)=0 \forall \quad u \in \text { domain } D_{\mathbf{A}} .
$$

Hence, $D_{\mathbf{A}}^{*} \mathbf{v}=0$, and we have an orthogonal decomposition

$$
\Omega_{1}^{0}=\left(\operatorname{range} D_{\mathbf{A}}\right) \oplus\left(\operatorname{ker} D_{\mathbf{A}}^{*}\right) .
$$

Since the adjoint of a semi-Fredholm operator is semi-Fredholm, it follows that $D_{\mathbf{A}}^{*}$ is semi-Fredholm. In particular $D_{\mathbf{A}}^{*} \mathbf{v}$ has closed range. If $u \in \Omega_{0}^{0}$ is orthogonal to $D_{\mathbf{A}}^{*} \mathbf{v}$ for all $\mathbf{v} \in$ domain $D_{\mathbf{A}}^{*}$, then $D_{\mathbf{A}} u=0$, and $u=0$. Thus, range $D_{\mathbf{A}}^{*}$ is dense in $\Omega_{0}^{0}$, which implies that $D_{\mathbf{A}}^{*}$ is surjective.

It follows from the decomposition (22) that the restriction of $D_{\mathbf{A}}^{*}$ to (range $D_{\mathbf{A}}$ ) $\cap\left(\right.$ domain $\left.D_{\mathbf{A}}^{*}\right)$ is surjective. Since $D_{\mathbf{A}}=d+S$ has domain $\Omega_{0}^{1}$, and $S$ maps $\Omega_{0}^{1}$ to $\Omega_{1}^{1}$,

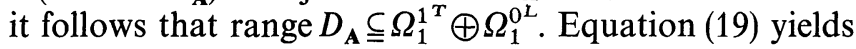

$$
\text { (range } \left.D_{\mathbf{A}}\right) \cap\left(\text { domain } D_{\mathbf{A}}^{*}\right) \subseteq \Omega_{1}^{1^{T}} \oplus \Omega_{1}^{1^{L}}=\Omega_{1}^{1}=\operatorname{domain} \operatorname{Div}_{\mathbf{A}} \text {. }
$$

Since the negative of $D_{\mathbf{A}}^{*}$ extends $\operatorname{Div}_{\mathbf{A}}$, Eq. (18), it follows that $\operatorname{Div}_{\mathbf{A}}$ is surjective which proves regularity of $\mathbf{J}^{-1}(0)$.

\section{Concluding Remarks}

In most papers on the physical content of Yang-Mills theory the singularities of the constraint set were ignored. The results presented here justify this approach as far as Yang-Mills fields in Minkowski space with square integrable Cauchy data are concerned. It should be noted that the assumption of square integrability is violated in the presence of symmetry breaking. Thus, the singularities of the constraint set in a symmetry breaking Yang-Mills-Higgs theory have to be studied separately.

\section{References}

1. Segal, I.: The Cauchy problem for the Yang-Mills equations. J. Funct. Anal. 33, 175-194 (1979)

2. Ginibre, J., Velo, G.: The Cauchy problem for coupled Yang-Mills and scalar fields in temporal gauge. Commun. Math. Phys. 82, 1-28 (1981)

3. Eardley, D., Moncrief, V.: The global existence of Yang-Mills-Higgs fields in 4-dimensional Minkowski space. I. Local existence and smoothness properties. Commun. Math. Phys. 83, 171-191 (1982)

4. Eardley, D., Moncrief, V.: The global existence of Yang-Mills-Higgs fields in 4-dimensional Minkowski space. II. Completion of proof. Commun. Math. Phys. 83, 193-212 (1982)

5. Moncrief, V.: Gribov degeneracies: coulomb gauge condition and initial value constraints. J. Math. Phys. 20, 579-585 (1979)

6. Binz, E., Sniatycki, J., Fischer, A.: Geometry of classical fields. Amsterdam: North-Holland 1988

7. Arms, J.: The structure of the solution set for the Yang-Mills equations. Math. Proc. Camb. Phil. Soc. 90, 361-372 (1981)

8. Arms, J., Marsden, J., Moncrief, V.: Symmetry and bifurcation of momentum mappings. Commun. Math. Phys. 78, 455-478 (1981)

9. Marsden, J., Weinstein, A.: Reduction of symplectic manifolds with symmetry. Rep. Math. Phys. 5, 121-130 (1974)

10. Kato, T.: Perturbation theory for linear operators, 2nd. (ed.). Berlin, Heidelberg, New York: Springer 1984

11. Adams, R.: Sobolev spaces. Orlando, Florida: Academic Press 1975 
